\title{
Effect of preoperative pregabalin on analgesia and interleukins after lumbotomy: prospective, randomized, comparative, double-blind study
}

This article was published in the following Dove Medical Press journal: Journal of Pain Research

\author{
Ana Ellen Queiroz Santiago \\ Plinio da Cunha Leal \\ Ed Carlos Rey Moura \\ Reinaldo Salomão \\ Milena Karina Coló \\ Brunialti \\ Rioko Kimiko Sakata
}

Department of Anesthesia, Universidade Federal de São Paulo, São Paulo, Brazil
Correspondence: Rioko Kimiko Sakata Universidade Federal de São Paulo, Rua Botucatu 593, Vila Clementino, São Paulo, SP, CEP 04023-062, Brazil

Tel/Fax +55 II 55764848

Email rsakata@unifesp.br
Background: Pregabalin is an anticonvulsant and has been used for postoperative analgesia. This study aimed to assess the effect of a single preoperative dose of pregabalin for analgesia after nephrectomy.

Methods: The study was prospective, randomized, comparative, and double-blinded, conducted in 40 kidney transplant donors, between 18 and 60 years, American Society of Anesthesia physical status I or II. Epidural anesthesia was performed with $15 \mathrm{~mL}$ of $0.5 \%$ ropivacaine single shot and general anesthesia with $3 \mu \mathrm{g} / \mathrm{kg}$ of fentanyl, propofol, atracurium, and sevoflurane, and $50 \%$ of oxygen without nitrous oxide. Patients in group 1 were administered $300 \mathrm{mg}$ of pregabalin and those in group 2 were administered placebo, in identical capsules, 1 hour prior to surgery. Postoperative analgesia was supplemented with tramadol. The following parameters were assessed: pain intensity after 6 and 24 hours; pain threshold, from the thenar and peri-incisional region, analgesic supplementation; ILs (IL6, IL8, and IL10) prior to surgery and after 6 and 24 hours. Results: The pain intensity was lower with pregabalin after 24 hours (G1:2.5 $\pm 2.4, \mathrm{G} 2: 3.0 \pm 2.6$ ). There was no difference in the sensitivity of the thenar and peri-incisional region after 6 and 24 hours; in the number of patients requiring supplementation ( $\mathrm{G} 1=15 \%, \mathrm{G} 2=45 \%)$; concentrations of IL-6, IL-8, and IL-10; and side effects (nausea, vomiting, dizziness, and pruritus).

Conclusion: Pregabalin in a single preoperative dose of $300 \mathrm{mg}$ reduced pain intensity 24 hours after lumbotomy.

Keywords: pregabalin, lumbotomy, postoperative analgesia, kidney transplant, nephrectomy

\section{Introduction}

Opioids are effective in controlling postoperative pain, but the use of large isolated doses of these drugs is associated with side effects, and multimodal treatment is recommended. ${ }^{1}$

Several drugs have been used for intra and postoperative analgesia. Pregabalin is a calcium channel modulator and acts to attenuate the hyperexcitability of neurons. ${ }^{2}$ Some studies have shown that pregabalin administered prior to surgery promotes the reduction of opioid consumption ${ }^{3-5}$ and decreases pain intensity and opioid side effects. ${ }^{4,5}$ However, in other studies there was no benefit of pregabalin. ${ }^{6,7}$

Administration of $300 \mathrm{mg}$ of pregabalin prior to surgery reduced opioid consumption after open transperitoneal nephrectomy ${ }^{8}$ as the use of $150 \mathrm{mg}$ of pregabalin on the day of laparoscopic nephrectomy and after 1 day. ${ }^{9}$

Inflammatory ILs such as IL-6 and IL-8 are released during the trauma causing inflammation and pain. ${ }^{10,11} \mathrm{IL}-10$, which is anti-inflammatory, is also released by trauma. ${ }^{11,12}$ 
It has been proposed that gabapentin could increase the expression of the anti-inflammatory cytokine IL-10 in the spinal cord, leading to the inhibition of proinflammatory cytokines such as tumor necrosis factor- $\alpha$ (TNF- $\alpha$ ), but also IL-1b and IL-6. ${ }^{13}$ The release of proinflammatory cytokines, including IL-1, IL-6, IL-8, and TNF- $\alpha$, is notably inhibited by IL-10. ${ }^{14}$

Due to the characteristics of pregabalin, its preoperative administration may promote analgesic effect and may decrease the inflammatory IL release, but the studies are conflicting about the benefit of reducing inflammatory ILs. Hence, this study was carried out with the purpose of assessing the pain control and the postoperative inflammatory reaction by measuring the inflammatory cytokines.

The primary objective of this study was to investigate the effect of single preoperative dose of pregabalin on pain intensity after nephrectomy. Secondary objectives were to assess pain pressure threshold with algometer and IL-6, IL-8, and IL10 concentrations.

The hypothesis of the study is that preoperative pregabalin promotes a decrease in pain intensity, and the total dose of opioid consumption, and in inflammatory cytokines.

\section{Materials and methods}

This study was prospective, randomized, comparative, and double-blind. There were no changes in methods after the eligibility criteria.

SPSS $17^{\circledR}$ statistical software was used to calculate the sample size. Based on the preliminary assessment, ${ }^{15}$ an SD of pain intensity score within the group of 2.2 was estimated. For the power of 0.95 and $\alpha=0.05$, the sample size calculated for each group was 20 patients.

Randomization was carried out by the software Research Randomizer (www.randomizer.org; ${ }^{\circledR} 1997-2016$ by Geoffrey C. Urbaniak and Scott Plous). The groups drawn were placed in envelopes with numbers. On the day of surgery, the envelope containing the number and the group to which the patient belonged to was removed. A nurse not involved with the protocol opened the envelope referring to this patient and administered pregabalin or placebo capsules. None of the other researchers involved in the work or data collection knew the group to which the patient belonged to.

After obtaining approval by the Ethics Committee of the Federal University of São Paulo (number 0774/10) and the written informed consent, this study was conducted in accordance with the Declaration of Helsinki. A total of 40 kidney donors, aged between 18 and 60 years, of both genders, physical status ASA I or II by the American Society of Anesthesia, submitted to nephrectomy by lumbotomy were included in the study. Lumbar incision is the usual approach for kidney donor, which permits access to the kidney (retroperitoneal) without entering the peritoneal cavity. Patients with chronic pain, psychiatric illness, drug user, and those who were using analgesic up to 2 weeks ago were excluded.

Participants in group 1 were administered $300 \mathrm{mg}$ of pregabalin and those in group 2 were administered placebo in four identical capsules 1 hour prior to surgery.

Epidural anesthesia was conducted with $15 \mathrm{~mL}$ of $0.5 \%$ ropivacaine single shot prior to the induction of general anesthesia with fentanyl ( $3 \mu \mathrm{g} / \mathrm{kg})$, propofol ( $2 \mathrm{mg} / \mathrm{kg})$, atracurium $(0.5 \mathrm{mg} / \mathrm{kg})$, and maintenance with sevoflurane and $50 \%$ of oxygen without nitrous oxide. Ondansetron (4 mg) was administered at the end of the procedure. After closure of the skin, sevoflurane was discontinued, and the neuromuscular blockade was antagonized.

Postoperative analgesia was supplemented with subcutaneous tramadol (100 mg), according to the patients' request and the dose was recorded.

The following parameters has been assessed: pain intensity by the numerical scale after 6 and 24 hours; dose of analgesic for 24 hours; pressure pain threshold with algometer at the thenar and peri-incisional region prior to surgery and after 24 hours; concentration of ILs (IL-6, IL-8, and IL-10) by the ELISA method, prior to surgery and after 6 and 24 hours. Side effects were noted.

The primary outcome was the reduction of pain intensity. The secondary outcomes were need of analgesic complementation, pain threshold, ILs, and adverse effects. No change in outcome was made after the trial commenced.

The analysis of the results was carried out by the software SPSS $17^{\circledR}$. The level of statistical significance was set at $\leq 0.05$. The tests used were Student's $t$-test for the duration of surgery, weight, age, height, pain intensity, and pain threshold; Fisher's exact test for ASA and side effects; chi-squared test for gender; Mann-Whitney $U$ test for total dose of tramadol and IL.

The study was registered in ClinicalTrials (number NCT01529190).

\section{Results}

The CONSORT flowchart is shown in Figure 1. There were no differences between groups regarding demographic data, physical status of patients and duration of surgery (Table 1). Intraoperative fentanyl was used only at induction in both the groups. Pain intensity was lower with pregabalin after 24 hours (Table 2). There was no significant difference in the number of patients requiring analgesic supplementation (G1: 15\%, G2: $45 \%$, Mann-Whitney $U$ test, $P=0.082$ ); nor in the dose of tramadol used in the postoperative period (G1: $0 ; 0-75$; 


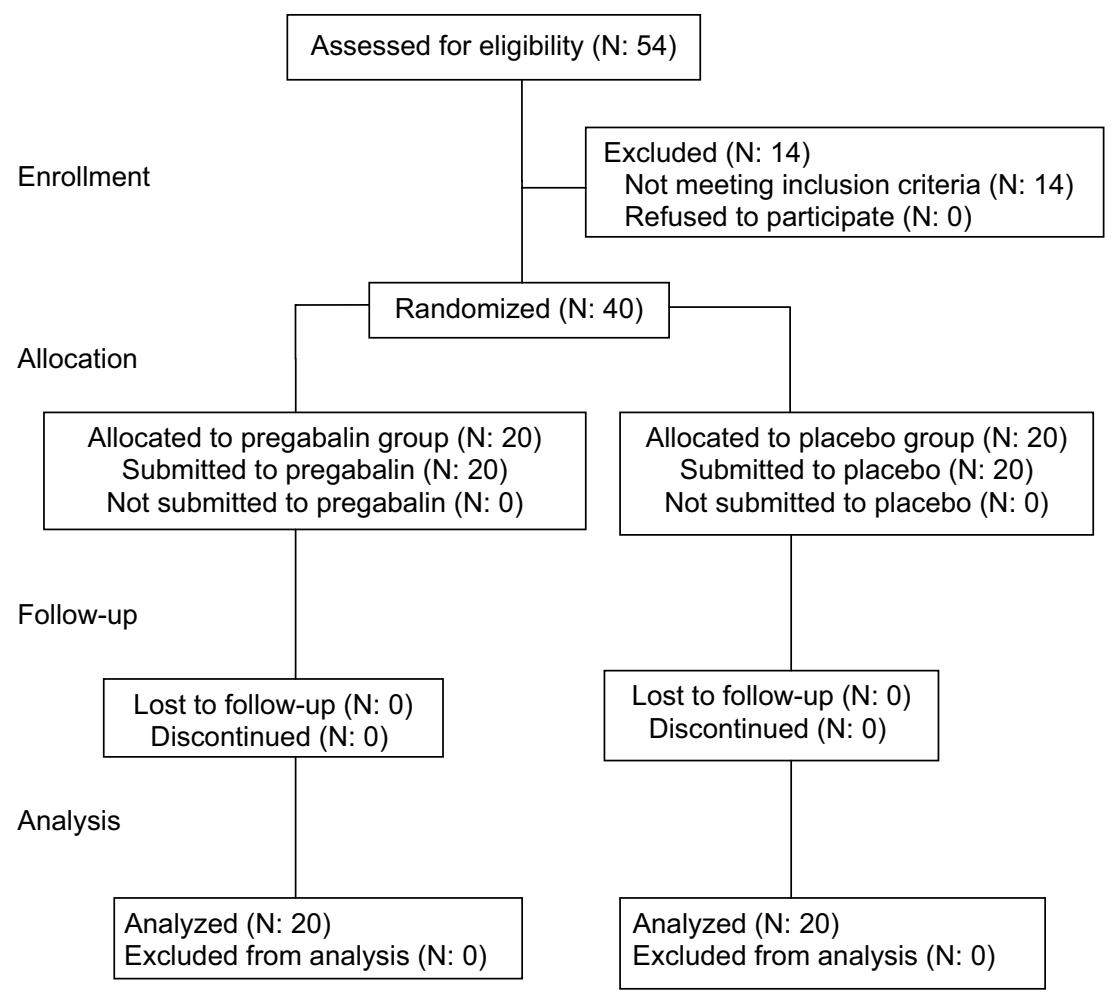

Figure I CONSORT flowchart.

Table I Demographic data - mean standard error, American Society of Anesthesia, duration of surgery

\begin{tabular}{|l|l|l|l|}
\hline Data & Pregabalin & Control & P-value \\
\hline Gender M:F & $\mathrm{II}: 9$ & $\mathrm{I} 2: 8$ & $\mathrm{I} .00^{\mathrm{a}}$ \\
\hline Age (years) & $45.0(\mathrm{I} .6)$ & $4 \mathrm{I} .0(\mathrm{I} .7)$ & $0.89^{\mathrm{b}}$ \\
\hline Weight $(\mathrm{kg})$ & $72.4(10.7)$ & $68.8(2.4)$ & $0.26^{\mathrm{b}}$ \\
\hline Height $(\mathrm{cm})$ & $167.5(\mathrm{I} .7)$ & $166.5(\mathrm{I} .3)$ & $0.70^{\mathrm{b}}$ \\
\hline ASA I:II & $16: 4$ & $16: 4$ & $1.00^{\mathrm{c}}$ \\
\hline Surgery duration & $108.8(6.7)$ & $108.3(8.6)$ & $0.96^{\mathrm{b}}$ \\
\hline
\end{tabular}

Notes: 'Chi-square test; 'bStudent's $t$-test; ' Fisher test.

Table 2 Pain intensity by numerical scale - mean (standard error), $\mathrm{Cl}$

\begin{tabular}{|l|l|l|l|}
\hline Times & Pregabalin & Control & $P$-value \\
\hline 6 hours & I.I (I.6) & $2.1(2.1)$ & 0.27 \\
$95 \% \mathrm{Cl}$ & $(0.3-1.9)$ & $(1.1-3.1)$ & \\
24 hours & $2.5 \pm 2.4$ & $3.0(2.6)$ & 0.04 \\
$95 \% \mathrm{Cl}$ & $(1.4-3.6)$ & $(1.7-4.2)$ & \\
\hline
\end{tabular}

Note: Student's $t$-test.

G2: 0; 0-100; median; 25-75 percentile; Mann-Whitney $U$ test; $P=0.580$ ). There was no difference between the groups in the pain threshold for pressure with algometer at the thenar and peri-incisional eminence (Table 3). There was no significant statistical difference between the groups in IL-6, IL-8, and IL-10 concentrations after 6 and 24 hours after surgical inci-
Table 3 Sensibility threshold evaluated with algometer - mean (standard error)

\begin{tabular}{|l|l|l|l|}
\hline Threshold & Pregabalin & Control & P-value \\
\hline Thenar & & & \\
$\quad$ Before incision & $6.4(1.9)$ & $7.6(1.9)$ & 0.06 \\
After 24 hours & $6.1(2.1)$ & $7.3(2.4)$ & 0.119 \\
\hline Peri-incisional & & & \\
Before incision & $5.5(1.9)$ & $5.9(2.1)$ & 0.599 \\
After 24 hours & $3.1(1.6)$ & $2.4(0.9)$ & 0.109 \\
\hline
\end{tabular}

Note: Student's t-test.

sion (Table 4). There were no significant differences in side effects (Table 5).

\section{Discussion}

In this study, a better analgesic effect was observed after 24 hours, with pregabalin being administered prior to lumbotomy, with no difference in the dose of supplementary analgesic, in the concentrations of ILs (IL-6, IL-8, and IL-10) and in the side effects.

Lumbotomy was chosen for the study because it causes intense stimulus of the pain pathway, and it is possible to assess the analgesic effect of the drug used.

Pain intensity was lower after 24 hours probably due to the reduction of central sensitization with pregabalin. Although 
Table 4 Plasma concentrations of IL-6, IL-8, and IL-I0 (pg/mL) median (minimum-maximum value)

\begin{tabular}{|c|c|c|c|}
\hline & Pregabalin $^{a}$ & Control & $P$-value ${ }^{a}$ \\
\hline \multicolumn{4}{|l|}{ IL-6 } \\
\hline $\begin{array}{l}\text { Preoperative } \\
6 \text { hours after } \\
\text { incision } \\
24 \text { hours after } \\
\text { surgery }\end{array}$ & $\begin{array}{l}0(0-12.5) \\
19.6(0-21.1) \\
21.8(0-110.4)\end{array}$ & $\begin{array}{l}0(0-37.6) \\
29(0-84.5) \\
27.4(0-68.7)\end{array}$ & $\begin{array}{l}0.62 \\
0.86 \\
0.90\end{array}$ \\
\hline \multicolumn{4}{|l|}{ IL-8 } \\
\hline $\begin{array}{l}\text { Preoperative } \\
6 \text { hours after } \\
\text { incision } \\
24 \text { hours after } \\
\text { surgery }\end{array}$ & $\begin{array}{l}0(0-107.5) \\
22.1(0-145.7) \\
18.6(0-120.5)\end{array}$ & $\begin{array}{l}0(0-189.8) \\
17.3(0-69.5) \\
0(0-168.8)\end{array}$ & $\begin{array}{l}0.46 \\
0.15 \\
0.41\end{array}$ \\
\hline \multicolumn{4}{|l|}{ IL-10 } \\
\hline $\begin{array}{l}\text { Preoperative } \\
6 \text { hours after } \\
\text { incision } \\
24 \text { hours after } \\
\text { surgery }\end{array}$ & $\begin{array}{l}0(0-19.2) \\
23.5(0-71) \\
2.6(0-107.5)\end{array}$ & $\begin{array}{l}0(0-108.4) \\
21.9(0-84.6) \\
21.9(0-90.5)\end{array}$ & $\begin{array}{l}0.89 \\
0.89 \\
0.61\end{array}$ \\
\hline
\end{tabular}

Note: a Mann-Whitney $U$ test.

Table 5 Side effects - number (\%)

\begin{tabular}{|l|l|l|l|}
\hline & Pregabalin & Control & P-value \\
\hline Nausea & $2(10 \%)$ & $6(30 \%)$ & 0.24 \\
Vomiting & $3(15 \%)$ & $4(20 \%)$ & 0.09 \\
Headache & $0(0 \%)$ & I $(5 \%)$ & 1.00 \\
Dizziness & I (5\%) & I (5\%) & 1.00 \\
Shaking & $0(0 \%)$ & I (5\%) & 1.00 \\
Pruritus & $0(0 \%)$ & I (5\%) & 1.00 \\
\hline
\end{tabular}

Note: Fisher's exact test.

there was no significant difference after 6 hours, the pain score was lower, the pain threshold in the peri-incisional region was higher after 24 hours, and the patients required a lower dose of complementation, showing its analgesic effect of pregabalin.

The lack of difference between groups in opioid consumption may be due to the access route used for nephrectomy. Another study showed a statistical difference between the groups in postoperative analgesic consumption, but different from this study, there was no difference in pain intensity. ${ }^{9}$

Pregabalin was used only prior to surgery. Administration of pregabalin also after surgery may promote better analgesic effect, since the increase in IL-6 persists for 10 days. However, the maintenance of pregabalin may cause side effects typical of this drug, such as dizziness, imbalance, and visual disturbance. Especially in elderly patients and locomotor procedures, this effect may compromise recovery. In addition, if pregabalin promotes desensitization of the pain pathway, relief may persist for a longer period. However, in metaanalysis, no better pain relief was observed with additional postoperative doses vs the single dose of pregabalin prior to the procedure. ${ }^{5} \mathrm{We}$, therefore, consider that postoperative doses are not required.

A quantity of $300 \mathrm{mg}$ was chosen, as a review showed that pregabalin promotes a greater reduction of opioid consumption with this dose. ${ }^{4}$ In one study, administration of $150 \mathrm{mg}$ of pregabalin prior to inducting anesthesia reduced pain scores and opioid consumption after hysterectomy. ${ }^{16}$ With pregabalin $150 \mathrm{mg}$ prior to myocardial revascularization surgery, there was a decrease in pain intensity after 4,12 , and 24 hours. ${ }^{17} \mathrm{~A}$ systematic review showed that a single preoperative dose of 100-300 mg was effective, with no difference between doses. ${ }^{5}$

Postoperative pain intensity can be evaluated by objective and subjective data, such as scales (such as numerical), threshold pressure, and need for postoperative analgesics. Dosage of substances (such as interleukins) and its variation contribute to the assessment of the inflammatory response, which may be exacerbated by pain intensity.

IL-6, IL-8 (inflammatory), and IL-10 (anti-inflammatory) were used, since they were the most important for assessment after surgery. The dosages of ILs were performed at the moments of greatest increase after surgical stimulus. Some authors have shown that the use of pregabalin leads to alteration of the chosen cytokines. ${ }^{13,14}$ One study showed that there is a decrease in inflammatory cytokines with the use of gabapentin prior to the painful stimulus. ${ }^{18}$

Plasma concentrations of IL-6 are detectable in $60 \mathrm{~min}-$ utes, peak between 4 and 6 hours and may persist for 10 days. ${ }^{11,19}$ IL-8 increases after 30-60 minutes, peak after 4 hours, and a progressive reduction in 24 hours. ${ }^{11,20}$ Peak of IL-10 is observed after 4-6 hours, returning to basal levels after 48 hours. ${ }^{12,21}$

Due to the plasma variation of the selected cytokines, we chose the 6-hour collection to observe their plasma peak and the 24-hour period to observe its regression and, in the end, to compare the two groups. In this study, ELISA was used, as in most studies. ${ }^{22-24}$

In this study, the IL-8 concentration was lower with pregabalin after 6 hours, although not significant. It may show some degree of protection against the surgical stimulus. Such fact may show some degree of protection against the surgical stimulus.

In this study, the IL-6 concentration was lower with pregabalin after 6 and 24 hours, although not significant. However, after 24 hours, IL-10 was lower and IL- 8 was higher in pregabalin group, also without statistical significance. 
Thus, pregabalin has not shown to be beneficial in reducing the inflammatory response.

The most common side effects of pregabalin in the perioperative period are sedation, dizziness, and visual disturbances. ${ }^{5,25}$ In this study, no difference was observed in the side effects of opioids. Although not significant, the incidence of the side effects of opioids (nausea and vomiting) was lower with pregabalin, probably due to the use of less supplementary opioid (pregabalin group used less tramadol in postoperative, but without statistical significance). Also, in other studies, these effects were less frequent. ${ }^{5}$ In one study, there was a higher incidence of visual disturbance. ${ }^{26}$ Dizziness, which is one of the most frequent side effects of pregabalin, ${ }^{5,27}$ was not different in both the groups. It may be due to its administration only before surgery.

Other common side effects of pregabalin such as sedation, drowsiness, visual disturbance, chronic headache, dry mouth, peripheral edema, incoordination, and ataxia ${ }^{5,25,27}$ were not observed in this study.

\section{Limitations}

Postoperative pain has a mixed mechanism, and it is possible that pregabalin is effective in reducing pain intensity in patients with a higher than inflammatory neuropathic pain component and further studies are needed.

\section{Conclusion}

Pregabalin (300 mg) before surgery reduces the pain intensity after 24 hours. There were no significant adverse events, demonstrating the safety of that dose. Therefore, pregabalin supported its efficacy as adjuvant for the management of postoperative pain, although it did not influence the plasma concentrations of interleukins.

\section{Data sharing statement}

The authors intend to share, if requested by the journal:

- All the deidentified participant individual data, in Excel tables

- The initial protocol in pdf

- Approval by the ethics committee in pdf

- Register of the study in pdf

These can be sent by means of attached files. These information is always available.

\section{Acknowledgment}

The authors acknowledge CAPES (Coordination of Improvement of Higher Education Personnel) for the support.

\section{Disclosure}

The authors report no conflicts of interest in this work.

\section{References}

1. Chou R, Gordon DB, de Leon-Casasola OA, et al. Management of postoperative pain: a clinical practice guideline from the American Pain Society, the American Society of Regional Anesthesia and Pain Medicine, and the American Society of Anesthesiologists' Committee on Regional Anesthesia, Executive Committee, and Administrative Council. J Pain. 2016;17(2):131-157.

2. Maitra S, Baidya DK, Bhattacharjee S, Som A. Perioperative gabapentin and pregabalin in cardiac surgery: a systematic review and metaanalysis. Rev Bras Anestesiol. 2017;67(3):294-304.

3. Vadivelu N, Mitra S, Narayan D. Recent advances in postoperative pain management. Yale J Biol Med. 2010;83(1):11-25.

4. Zhang J, Ho KY, Wang Y. Efficacy of pregabalin in acute postoperative pain: a meta-analysis. Br J Anaesth. 2011;106(4):454-462.

5. Mishriky BM, Waldron NH, Habib AS. Impact of pregabalin on acute and persistent postoperative pain: a systematic review and meta-analysis. Br J Anaesth. 2015;114(1):10-31.

6. Paech MJ, Goy R, Chua S, Scott K, Christmas T, Doherty DA. A randomized, placebo-controlled trial of preoperative oral pregabalin for postoperative pain relief after minor gynecological surgery. Anesth Analg. 2007;105(5):1449-1453.

7. Chang SH, Lee HW, Kim HK, Kim SH, Kim DK. An evaluation of perioperative pregabalin for prevention and attenuation of postoperative shoulder pain after laparoscopic cholecystectomy. Anesth Analg. 2009;109(4):1284-1286.

8. Bornemann-Cimenti H, Lederer AJ, Wejbora M, et al. Preoperative pregabalin administration significantly reduces postoperative opioid consumption and mechanical hyperalgesia after transperitoneal nephrectomy. Br J Anaesth. 2012;108(5):845-849.

9. Myhre M, Romundstad L, Stubhaug A. Pregabalin reduces opioid consumption and hyperalgesia but not pain intensity after laparoscopic donor nephrectomy. Acta Anaesthesiol Scand. 2017;61(10): $1314-1324$.

10. Watt DG, Horgan PG, Mcmillan DC. Routine clinical markers of the magnitude of the systemic inflammatory response after elective operation: a systematic review. Surgery. 2015;157(2):362-380.

11. Tsilimigras DI, Sigala F, Karaolanis G, et al. Cytokines as biomarkers of inflammatory response after open versus endovascular repair of abdominal aortic aneurysms: a systematic review. Acta Pharmacol Sin. 2018;39(7):1164-1175.

12. Nicholson G, Woodfine J, Bryant AE, et al. The hormonal and inflammatory responses to pelvic reconstructive surgery following major trauma. Injury. 2005;36(2):303-309.

13. Kremer M, Yalcin I, Nexon L, et al. The antiallodynic action of pregabalin in neuropathic pain is independent from the opioid system. Mol Pain. 2016;12:174480691663347.

14. XuYJ, Chen WK, Zhu Y, Wang SL, Miao CH. Effect of thoracic epidural anaesthesia on serum vascular endothelial growth factor $\mathrm{C}$ and cytokines in patients undergoing anaesthesia and surgery for colon cancer. $\mathrm{Br} J$ Anaesth. 2014;113(Suppl 1):i49-i55.

15. Agarwal A, Gautam S, Gupta D, Agarwal S, Singh PK, Singh U. Evaluation of a single preoperative dose of pregabalin for attenuation of postoperative pain after laparoscopic cholecystectomy. Br JAnaesth. 2008;101(5):700-704.

16. Jo HR, Chae YK, Kim YH, et al. Remifentanil-induced pronociceptive effect and its prevention with pregabalin. Korean $J$ Anesthesiol. 2011;60(3):198-204.

17. Ziyaeifard M, Mehrabanian MJ, Faritus SZ, et al. Premedication with oral pregabalin for the prevention of acute postsurgical pain in coronary artery bypass surgery. Anesth Pain Med. 2015;5(1): e24837. 
18. Dias JM, de Brito TV, de Aguiar Magalhães D, et al. Gabapentin, a synthetic analogue of gamma aminobutyric acid, reverses systemic acute inflammation and oxidative stress in mice. Inflammation. 2014;37(5):1826-1836.

19. Gebhard F, Pfetsch H, Steinbach G, Strecker W, Kinzl L, Brückner UB. Is interleukin 6 an early marker of injury severity following major trauma in humans? Arch Surg. 2000;135(3):291-295.

20. Liener UC, Brückner UB, Knöferl MW, Steinbach G, Kinzl L, Gebhard F. Chemokine activation within 24 hours after blunt accident trauma. Shock. 2002;17(3):169-172.

21. Iyer SS, Cheng G. Role of interleukin 10 transcriptional regulation in inflammation and autoimmune disease. Crit Rev Immunol. 2012;32(1):23-63.

22. Uçeyler N, Häuser W, Sommer C. Systematic review with meta-analysis: cytokines in fibromyalgia syndrome. BMC Musculoskelet Disord. 2011;12:245.
23. Leal PC, Salomão R, Brunialti MK, Sakata RK. Evaluation of the effect of ketamine on remifentanil-induced hyperalgesia: a double-blind, randomized study. J Clin Anesth. 2015;27(4):331-337.

24. Oliveira CM, Sakata RK, Slullitel A, Salomão R, Lanchote VL, Issy AM. Effect of intraoperative intravenous lidocaine on pain and plasma interleukin-6 in patients undergoing hysterectomy. Rev Bras Anestesiol. 2015;65(2):92-98.

25. Lam DM, Choi SW, Wong SS, Irwin MG, Cheung CW. Efficacy of pregabalin in acute postoperative pain under different surgical categories. Medicine. 2015;94(46):e1944.

26. Zhang J, Ho KY, Wang Y. Efficacy of pregabalin in acute postoperative pain: a meta-analysis. Br J Anaesth. 2011;106(4):454-462.

27. Verma V, Singh N, Jaggi A. Pregabalin in neuropathic pain: evidences and possible mechanisms. Curr Neuropharmacol. 2014;12(1):44-56.

\section{Publish your work in this journal}

The Journal of Pain Research is an international, peer reviewed, open access, online journal that welcomes laboratory and clinical findings in the fields of pain research and the prevention and management of pain. Original research, reviews, symposium reports, hypothesis formation and commentaries are all considered for publication.

\section{Dovepress}

The manuscript management system is completely online and includes a very quick and fair peer-review system, which is all easy to use. Visit http://www.dovepress.com/testimonials.php to read real quotes from published authors. 